\title{
Useful Energy Prediction Model of a Lithium-ion Cell Operating on Various Duty Cycles
}

This paper was downloaded from TechRxiv (https://www.techrxiv.org).

\section{LICENSE}

CC BY 4.0

SUBMISSION DATE / POSTED DATE

$12-10-2021 / 26-10-2021$

CITATION

Burzyński, Damian (2021): Useful Energy Prediction Model of a Lithium-ion Cell Operating on Various Duty Cycles. TechRxiv. Preprint. https://doi.org/10.36227/techrxiv.16799587.v1

$\mathrm{DOI}$

10.36227/techrxiv.16799587.v1 


\title{
Useful Energy Prediction Model of a Lithium-ion Cell Operating on Various Duty Cycles
}

\author{
Damian Burzyński
}

\begin{abstract}
The paper deals with the subject of the prediction of useful energy during the cycling of a lithium-ion cell (LIC), using machine learning-based techniques. It was demonstrated that depending on the combination of cycling parameters, the useful energy $\left(R U E_{c}\right)$ that can be transfered during a full cycle is variable, and also three different types of evolution of changes in $R U E_{c}$ were identified. The paper presents a new non-parametric $R U E_{c}$ prediction model based on Gaussian process regression. It was proven that the proposed methodology enables the $R U E_{c}$ prediction for LICs discharged, above the depth of discharge, at a level of $70 \%$ with an acceptable error, which is confirmed for new load profiles. Furthermore, techniques associated with explainable artificial intelligence were applied, for the first time, to determine the significance of model input parameters - the variable importance method - and to determine the quantitative effect of individual model parameters (their reciprocal interaction) on $R U E_{c}$ - the accumulated local effects model of the first and second order. Not only is the $R U E_{c}$ prediction methodology presented in the paper characterised by high prediction accuracy when using small learning datasets, but it also shows high application potential in all kinds of battery management systems.
\end{abstract}

Index Terms - cycle life modelling, Gaussian mixture model, lithium-ion battery, machine learning, predictive models, useful energy prediction.

\section{INTRODUCTION}

$\mathrm{I}_{\mathrm{th}}^{\mathrm{N}}$ $\mathrm{N}$ recent years, it is possible to observe a rapid increase in the demand for equipment designed for electric energy storage. At present, it is estimated that the highest increase refers to the industry branch related to electromobility, where solutions based on lithium-ion batteries are currently dominant. It is predicted that by the end of 2030, the demand for batteries will increase ten times in this sector alone [1]-[2]. The prevailing share of lithium-ion batteries on the market follows primarily from their ability to transfer energy quickly and effectively, whereby this ability is successively increased owing to the dynamic development of electrochemical battery technology [3].

The key issue related to the operation of these storages is

"This work was supported by the Polish Government under Grant Subwencja Badawcza 0212/SBAD/0512."

D. Burzyński is with the Poznan University of Technology, Faculty of Control, Robotics and Electrical Engineering, Piotrowo 3A, 60-965, Poznan, Poland (e-mail: damian.burzynski@ put.poznan.pl). their life. Degradation of lithium-ion cells (LICs) is a consequence of aging processes which take place during cycling, and also during the storage period [4]-[5]. Out of more than a dozen identified aging phenomena, lithium plating, formation, evolution and dissolution of the solid electrolyte interface (SEI), as well as electrolyte decomposition, particle gassing and corrosion of the current collectors are the pre-dominant contributors to the degradation process [6]. Without interference in the LIC structure, the effects of the aging processes may be determined through the loss in charge throughput and the increase in internal resistance (or impedance).

\section{A. RELATED PAPERS}

Based on the current state of knowledge, it can be concluded that in the case of LICs, usually separate degradation models are developed for cycling (cycle life) and storage period (calendar aging). Degradation models during cycling are multi-parameter models - their parameters include: values of discharge/charge current $\left(I_{d} / I_{c h}\right)$, depth of discharge $(D o D)$, cell temperature $\left(T_{c}\right)$ or ambient temperature, as well as the charge or energy throughput (expressed most frequently as the number of full equivalent cycles - FECs). At this point, it must be emphasised that some of these parameters are correlated with each other and their effect on the amount of energy throughput of LICs is non-linear [7]. The abovementioned facts make analysing and predicting the LICs' ability to transfer energy over the period of their lifetime highly complicated and require advanced methods. Regardless of the chemical composition of the cells, testing them is very time consuming. For this reason a more and more frequent practice is to conduct the testing under conditions which result in their accelerated degradation (e.g. under an increased load or at extreme temperatures), and then to predict their operational parameters under the assumed operating conditions [8]-[9].

A frequent approach among researchers is the use of methods based on regression [10]-[11]. In such models, in the majority of cases, the effect of selected cycling parameters on LIC degradation is analysed. Zhang et al. [12] developed a model which enables prediction of the remaining useful life of a battery (RUL) for a $\mathrm{LiNiCOAlO}_{2}$ cell. The tests were conducted under conditions of accelerated aging - at discharge currents $I_{d}=1 \mathrm{C}$ and $I_{d}=2 \mathrm{C}$ and at ambient temperatures 
$T_{a}=25^{\circ} \mathrm{C}$ and $T_{a}=40^{\circ} \mathrm{C}$. In paper [13] Dessaint et al. implemented two multi-parametric models which allow for the determination of the maximum number of cycles which may be carried out by $\mathrm{LiNiMnCoO}_{2}$ and $\mathrm{LiFePO}_{4}$ cells, depending on the actual cycling parameters $\left(I_{d}, I_{c h}, D o D, T_{a}\right)$.

In turn, Saha et al. in [14] used electrochemical impedance spectroscopy (EIS) and the Bayesian statistical approach to develop a new RUL method.

Moreover, as methods based on machine intelligence are being developed, it is possible to observe their more and more frequent use in problems related to the prediction of operational parameters of LICs such as, e.g. remaining useful life (RUL) [15]-[16] or capacity [17]. These methods are strictly based on data which are usually acquired from manymonths of experimental procedures [18]-[21]. In [22], Hannan et al. proposed the use of a deep neural network to determine the $\mathrm{SoC}$ for $\mathrm{LiNiMnCoO}_{2}$ cells. $\mathrm{Li}$ et al. [23] developed a method for estimating the capacity of $\mathrm{LiFePO}_{4}$ cells, based on a convolutional neural network. Moreover, the use of support vector machines was proposed in papers by [24] to predict the state of health $(\mathrm{SoH})$ of batteries. In the past, approaches based on sample entropy [25], fuzzy logic [26] and RaoBlackwellization particle filter [27] have also been used to determine the RUL.

The GPR technique used in that paper had previously been used to predict the capacity fade or RUL. In [28], Howey et al. developed a model to predict the capacity fade of a $\mathrm{LiCoO}_{2}$ cell under variable load and temperature conditions. On the other hand in [29] Hariharan et al. used the deep GPR to estimate the end of life $(E o L)$ - the chemical composition of the cell was not specified. GPR was also used to predict battery degradation during calendar aging, and this issue was touched upon in paper by [30].

Because of the fact that, for some designers of systems powered from cells, $\mathrm{SoH}$ is not always a sufficient assessment indicator, other methods which enable the determination and prediction of the available power and energy in LICs have been developed recently. In [31], an attempt was made to predict the energy available in the battery for two cells LiNiMnCoO ${ }_{2}$ and $\mathrm{LiFePO}_{4}$, using particle filtering. In a paper by [32], the state of the available energy at different $I_{d}$ and at different $T_{a}$, taking into account degradation, was determined for a $\mathrm{Li}_{4} \mathrm{Ti}_{5} \mathrm{O}_{12}$ cell using a circuit model.

\section{B. KEY CONTRIBUTIONS}

Although many different approaches have been developed to date in the aspect of modelling the effects of aging phenomena occurring in LICs, in particular the prediction of available energy and power, this issue has not yet been sufficiently explored. In many applications, not only is knowledge of the predicted useful energy of the LIC over its lifetime required, but also of the predicted changes in degradation trends. For this reason, the following contributions have been made in this paper:

1) A major contribution is the development of a model based on machine learning, which enables the prediction of the useful energy which a LIC is able to transfer during a

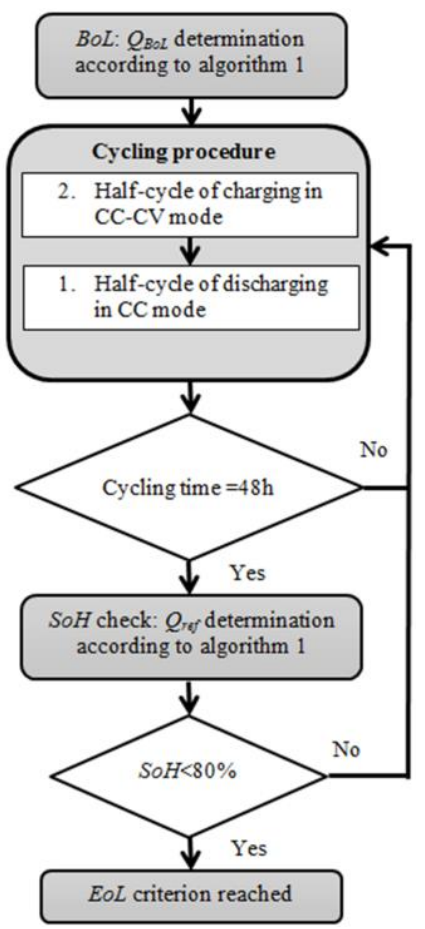

Fig. 1. Block diagram of the experimental procedure.

single duty cycle from begin of life $(B o L)$ to end of life $(E o L)$. The proposed model has a new structure of input parameters consideration of the past energy throughput of LICs as a model parameter is an improvement of the currently existing methods.

2) The result of this research is a new dataset that can be used to test current methods and develop new predictive methods for the useful energy or $\mathrm{SoH}$ of LICs.

3) A demonstration of the variability of $R U E_{c}$ depending on the combination of cycling parameters, and also identification of three types of evolution of changes in $R U E_{c}$ in the period of operation of the LIC under consideration.

4) For the first time, the following techniques have been used: explainable artificial intelligence (XAI) - variable importance $(\mathrm{VI})$ - to determine the significance of parameters of the GPR model, and accumulated local effects (ALE) - to determine their quantitative effect on $R U E_{c}$.

\section{STRUCTURE OF THE PAPER}

The remaining part of the paper is organised as follows. Chapter II outlines the procedure for obtaining experimental data and determining the useful energy $R U E_{\mathrm{c}}$ and also describes the results of preliminary analyses and calculations. Chapter III presents the applied methodology, GPR and XAI techniques. The results obtained and the discussion of the verification procedure are included in chapter IV.

\section{DATA PREPARATION}

In order to obtain data related to the ability of LICs to transfer energy during cycling, they were subjected to aging tests under various load and temperature conditions. Commercial Samsung 18650 LICs (cylindrical), with a nominal capacity of $2600 \mathrm{mAh}$ and nominal voltage of $3.63 \mathrm{~V}$ were selected for the tests. The cathode material of the tested 


\begin{tabular}{|c|c|c|c|c|c|c|c|c|c|}
\hline Cell ID & $\begin{array}{l}T_{c} \\
\text { (C) }\end{array}$ & $\begin{array}{l}I_{d} \\
\text { (A) }\end{array}$ & $\begin{array}{l}I_{\text {ch ang }} \\
\text { (A) }\end{array}$ & $\begin{array}{l}D \circ D \\
(\%)\end{array}$ & $\begin{array}{c}F E C_{\text {tot }} \\
(-)\end{array}$ & $\begin{array}{c}R U E_{c} \text { at } \\
B o L \\
(-)\end{array}$ & $\begin{array}{c}R U E_{c} \text { at } \\
E o L \\
(-)\end{array}$ & $\begin{array}{c}\triangle R U E_{c} \\
(\%)\end{array}$ & $\begin{array}{l}E_{t h} \text { tot } \\
(\mathrm{Wh})\end{array}$ \\
\hline H5 & 18.9 & -2.6 & 2.08 & 100 & 83 & 0.872 & 0.687 & 18.5 & 2302 \\
\hline $\mathrm{A} 31$ & 29 & -7.8 & 2.62 & 100 & 184 & 0.827 & 0.418 & 40.9 & 3451 \\
\hline D11 & 17.8 & -2.6 & 2.47 & 50 & 183 & 0.545 & 0.545 & 0 & 3435 \\
\hline B37 & 20.9 & -2.6 & 2.35 & 100 & 72 & 0.872 & 0.709 & 16.3 & 1516 \\
\hline $\mathrm{A} 30$ & 17.8 & -2.6 & 2.4 & 73 & 149 & 0.776 & 0.663 & 11.3 & 2753 \\
\hline $\mathrm{H} 2$ & 16.6 & -2.6 & 1.8 & 27 & 275 & 0.304 & 0.304 & 0 & 5029 \\
\hline $\mathrm{A} 36$ & 25.1 & -5.2 & 2.09 & 50 & 426 & 0.536 & 0.536 & 0 & 8002 \\
\hline D14 & 23.9 & -2.6 & 2.15 & 100 & 130 & 0.88 & 0.74 & 14 & 2517 \\
\hline B40 & 30.3 & -5.2 & 2.2 & 100 & 1054 & 0.875 & 0.55 & 32.5 & 19804 \\
\hline A 35 & 32.5 & -7.8 & 2.52 & 100 & 664 & 0.865 & 0.33 & 53.5 & 12449 \\
\hline A 20 & 25.7 & -5.2 & 1.81 & 100 & 209 & 0.942 & 0.704 & 23.8 & 3933 \\
\hline $\mathrm{C} 33$ & 32.7 & -2.6 & 3.04 & 74 & 306 & 0.789 & 0.749 & 4 & 5765 \\
\hline H10 & 33 & -2.6 & 2.78 & 74 & 344 & 0.795 & 0.759 & 3.6 & 6478 \\
\hline H9 & 35.3 & -2.6 & 2.85 & 100 & 226 & 0.945 & 0.752 & 19.3 & 4254 \\
\hline D16 & 43.2 & -7.8 & 2.64 & 100 & 905 & 0.911 & 0.294 & 61.7 & 16978 \\
\hline B32 & 39.7 & -5.2 & 2.66 & 100 & 676 & 0.922 & 0.672 & 25 & 13573 \\
\hline D1 & 33.2 & -5.2 & 2.38 & 16 & 3984 & 0.165 & 0.165 & 0 & 74901 \\
\hline A 24 & 36.6 & -5.2 & 2.18 & 50 & 1721 & 0.546 & 0.546 & 0 & 32340 \\
\hline D4 & 29.2 & -2.6 & 1.2 & 100 & 1908 & 0.982 & 0.786 & 19.6 & 36109 \\
\hline $\mathrm{C} 26$ & 36.4 & -5.2 & 1.9 & 100 & 909 & 0.97 & 0.689 & 28.1 & 17083 \\
\hline A 23 & 36.6 & -7.8 & 1.74 & 100 & 557 & 0.939 & 0.221 & 71.8 & 10458 \\
\hline H12 & 58.6 & -7.8 & 2.68 & 100 & 587 & 0.931 & 0.533 & 39.8 & 10993 \\
\hline H1 & 53.8 & -7.8 & 1.88 & 100 & 563 & 1.005 & 0.504 & 50.1 & 10555 \\
\hline D6 & 50.1 & -2.6 & 3.46 & 100 & 375 & 1.046 & 0.813 & 23.3 & 7048 \\
\hline B35 & 47.5 & -2.6 & 2.37 & 100 & 702 & 1.057 & 0.848 & 20.9 & 13206 \\
\hline A38 & 52.5 & -5.2 & 2.54 & 100 & 960 & 1.002 & 0.717 & 28.5 & 18034 \\
\hline H13 & 62.1 & -7.8 & 3.11 & 75 & 716 & 0.744 & 0.564 & 18 & 13409 \\
\hline $\mathrm{C} 10$ & 32.6 & -2.6 & 2.61 & 100 & 1460 & 0.995 & 0.798 & 19.7 & 27446 \\
\hline $\mathrm{D} 2$ & 33.7 & -2.6 & 3.2 & 100 & 160 & 0.934 & 0.742 & 19.2 & 3028 \\
\hline
\end{tabular}

cells was composed of $\mathrm{LiNi}_{0.33} \mathrm{Mn}_{0.33} \mathrm{Co}_{0.33} \mathrm{O}_{2}$ compound, while the anode was made of graphite.

\section{A. EXPERIMENTAL PROCEDURE}

The procedure presented in Fig. 1 was adopted for the purpose of performing aging tests. Before starting the aging procedure of each cell, their reference charge $\left(Q_{\text {ref }}\right)$ was determined in accordance with algorithm 1 .

Then, each of the cells was subjected to cycling under unique temperature and load conditions. During the tests, a Panasonic MIR-254 temperature chamber was used to maintain a constant ambient temperature. Momentary temperatures of cells were recorded with probes located in central points of the cells. The average charge current from all half-cycles completed from $\mathrm{BoL}(\mathrm{SoH}=100 \%)$ to $\mathrm{EoL}(\mathrm{SoH}=80 \%)$ was adopted as the value of charge current during analyses. During the experimental procedures, LICs were kept in the voltage operating window specified by the manufacturer, i.e. from $2.75 \mathrm{~V}\left(U_{d s c h}\right)$ to $4.2 \mathrm{~V}\left(U_{c h}\right)$. Several $D o D$ levels were selected ranging between $16 \%$ and $100 \%$, with an assumption that $D o D$ is the charge which can be obtained in the given combination of cycling parameters. For example $D o D=100 \%$ corresponds to a discharge in the full voltage operating window. The cell cycling process was controlled by a Cadex C8000 tester dedicated to electrochemical cells (values of momentary currents, voltages and temperatures of LICs were measured using separate wires). To sum up, 29 cells in total were tested at 4 different $T_{a}\left(10^{\circ} \mathrm{C}, 15^{\circ} \mathrm{C}, 25^{\circ} \mathrm{C}\right.$ and $\left.40^{\circ} \mathrm{C}\right)$, at 3 different values of $I_{d}(-2.6 \mathrm{~A},-5.2 \mathrm{~A}$ and $-7.8 \mathrm{~A})$ and at 5 different $D o D$ levels $(16 \%, 27 \%, 50 \%, 75 \%$ and $100 \%)$. The average value of charge current $\left(I_{\text {ch_avg }}\right)$ ranged between $1.2 \mathrm{~A}$ and 3.46 A. Table I contains detailed values of the cycling parameters adopted during the aging tests.

The condition for completion of each aging procedure was the degradation of the cell to $\mathrm{SoH}=80 \%$. The actual $\mathrm{SoH}$ of the cells was checked every 48 hours under reference conditions (described in algorithm 1) using the following relationship (1)

$$
S o H=\frac{Q_{r e f}}{Q_{B o L}} \cdot 100 \%
$$

where $Q_{\text {ref }}$ is the collected charge, $Q_{B o L}$ is the charge collected before the start of the LIC cycling.

\section{B. USEFUL ENERGY DETERMINATION}

In order to determine the useful energy throughput of a LIC during a single duty cycle over its entire life, the following methodology was adopted:

1) The assumption that one complete duty cycle consists of a discharge half-cycle and the immediately following charge half-cycle.

2) Calculation of the charge throughput during a single duty cycle (collected during discharge $Q_{d s c h}$ and charge $Q_{c h}$ ) using the Coulomb Counting method, on the basis of equation (2).

Determination of the relative useful energy $R U E_{c}$ throughput during the duty cycle in accordance with relationship (3). $R U E_{c}=1$ means that a cell transfered nominal energy (18.876 Wh) during a full duty cycle (a discharge followed by a charge). 


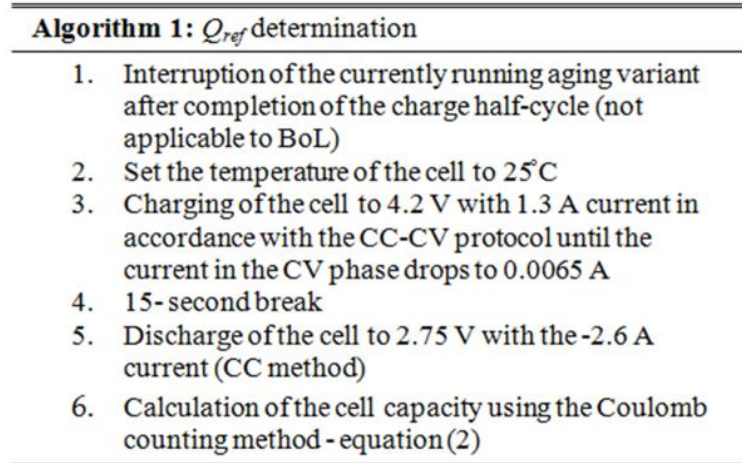

3) After each control procedure, determination of the number of completed full equivalent duty cycles (FECS) for the needs of the prediction model.

4) Determination of $F E C_{t o t}$ as the sum of RUEc from all completed LIC duty cycles from BoL to EoL. An assumption was made that the transfer of nominal energy $(18.876 \mathrm{Wh})$ during two consecutive charge and discharge half-cycles constitutes a full equivalent cycle $F E C$.

5) Determination of the total energy throughput $E_{\text {th_tot }}$ as a product of total charge throughput of LIC, multiplied by its voltage on terminals.

$$
\begin{gathered}
Q_{c h / d s c h}=Q_{0}+\int_{0}^{t} I_{c h / d s c h}(t) d t \\
R U E_{c}=\frac{Q_{d s c h} \cdot U_{\text {ave_dsch }}+Q_{c h} \cdot U_{\text {ave_ch }}}{2 \cdot C_{n o m} \cdot U_{n o m}}
\end{gathered}
$$

where $Q_{0}$ is the initial charge accumulated in a LIC, $U_{\text {ave_dsch }}$ is an average voltage during a discharge half-cycle, $U_{\text {ave_ch }}$ is an average voltage during a charge half-cycle, $C_{n o m}$ is the nominal capacity and $U_{\text {nom }}$ is the nominal voltage.

\section{INITIAL ANALYSIS AND CALCULATIONS}

The tests carried out showed that for the LICs discharged to $D o D>70 \%$ the value of the transferred $R U E_{c}$ is variable during their considered life. In the case of variants with $D o D<70 \%$, LICs transferred the fixed energy throughout their life $\left(R U E_{c}=\right.$ const $)$. Depending on the combination of the values of the cycling parameters, three types of evolution of $R U E_{c}$ changes were observed during the analysed period of operation: a) approximately linear, b) a slow decrease in the first phase of life followed by a phase of accelerated loss of

energy transfer, c) a characteristic inflection point occurred after the phase of releasing the assumed charge, followed by a phase of rapid loss of energy transfer. The phenomena described in points b) and c) were present in cells "A23", "D14", "D16", "A30", "H10" and "C33". The selected characteristics of the $R U E_{c}$ of cells, in which the effect described above occurred, are presented in Fig. 2, and the results of the remaining experimental measurements of the cells, are available online (raw data: https://data.mendeley.com/datasets/fzp5wx28kw/1).

For each completed aging variant, it was checked how the ability of the cell to transfer $R U E_{c}$ decreased. For this purpose, the $\triangle R U E_{c}$ difference (eq. 4) between $R U E_{c}$ determined at

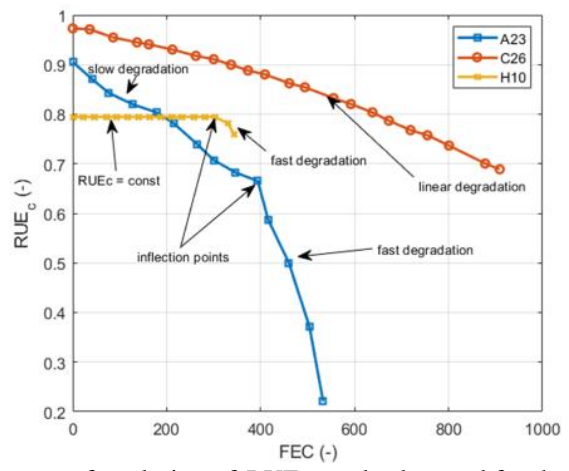

Fig. 2. Three types of evolution of $R U E_{c}$ trends observed for the tested LIC. $B o L$ and $E o L$ was calculated.

$$
\Delta R U E_{c}=\left(R U E_{c_{-} B o L}-R U E_{c_{-} E o L}\right) \cdot 100 \%
$$

Depending on the conditions under which the LICs were cycled, $\triangle R U E_{c}$ reached values exceeding $50 \%$ (cells: "A35", "D16", "H1" and "H23") which means that in the period preceding EoL the cells were not capable of transfering even half of the initial $R U E_{c}$. A high value of $\triangle R U E_{c}$ was observed for cells discharged with a high current $(-7.8 \mathrm{~A})$ regardless of the temperature conditions (average cyclic temperature of the cell). The lowest values of $\triangle R U E_{c}$ occurred for cycled cells at incomplete $D o D$ ("A30", "C33", "H10" and "H13").

Additionally, $F E C_{\text {tot }}$ and $E_{\text {th_tot }}$ completed by each LIC were determined and listed for information purposes in Table I. Results of initial calculations are included in Table I.

\section{PROPOSED METHODOLOGY}

\section{A. PRoposed MODEL}

The amount of $R U E_{c}$ which LICs are capable of transfering during a full duty cycle over their lifetime depends on the combination of values of cyclic operating parameters and the number of completed FECs. As the tests demonstrated, LICs may have a similar ability to transfer $R U E_{c}$ even if they have completed a significantly different number of FECS. One additional issue is the fact that the cyclic operating parameters of a LIC are strongly correlated. For instance higher values of $D o D, I_{d}$ and $I_{c h \_a v g}$ result in an increase in the average temperature of the LIC, which, in turn, determines the amount of $R U E_{c}$. Furthermore, the impossibility of separating the impact of the above-mentioned parameters during a duty cycle makes it highly difficult to determine the precise effect of individual parameters on the amount of $R U E_{c}$. In this paper, in order to develop a prediction model for the relative useful energy $R U E_{c}$ the framework presented in Fig. 3 was adopted. Taking the above-mentioned challenges into account, the author proposes the use of the non-parametric model, whose structure strictly matches the form of learning data. This model belongs to the group of machine learning models - it is more frequently referred to as GPR and is a non-parametric regression technique based on Gauss processes. The use of a non-parametric model forces out the use of techniques which enable the interpretation of the manner in which the implemented non-parametric model operations. 


\section{Data preparation}

1. Obtain the data from aging procedures of $29 \mathrm{LICs}$

2. Determination of characteristic model parameters $R U E_{c}$ and $F E C$

3. Data pre-processing (normalization)

Prediction model development

1. Selection of kernel and basis functions

2. Training the model

3. Model verification

\section{Model explanation}

1. Determination of variable importance

2. Determination of first- and second-order ALE for model variables

Fig 3. General flowchart of the applied framework.

\section{B. GAUSSIAN PROCESS REGRESSION}

Gaussian process regression (GPR) belongs to the group of kernel-based non-parametric models [33]-[34]. In this model, the Gaussian probability distribution is defined for each finite set of input variables $x_{i}$.

$$
f(x) \sim G P\left(\mu(x), \operatorname{cov}\left(x, x^{\prime}\right)\right)
$$

where $\mu(x)$ is mean function, and $\operatorname{cov}\left(x, x^{\prime}\right)$ is the covariance function (kernel function).

The kernel functions contain parameters related to the scaling of responses of model $y$ and input vector $x$ and are referred to as hyperparameters $\theta$. For the majority of standard kernels, the hyperparameters include: standard deviation $\sigma_{f}$ and characteristic length $\sigma_{l}$.

The matching of the structure of the model to the learning set takes place by defining, for each sample of the learning dataset $x_{i}$, a function $f\left(x_{i}\right)$ having the Gaussian distribution - hereafter called a hidden variable - and a set of basis functions $h(x)$, which transfer the input vector $x_{i}$ from the original feature space $R^{d}$ to the extended feature space $R^{p}$.

For the needs of the $R U E_{c}$ prediction, the following structure of the model has been adopted

$$
y(x)=h(x)^{T} w+f(x)+\varepsilon
$$

where $h(x)$ is the set of basis functions, $w$ is the vector of basis function coefficients, $\varepsilon$ is noise with normal distribution.

Model prediction $y$ takes place on the basis of input vector $x$, and the learning dataset

$$
P\left(y_{i} \mid f\left(x_{i}\right), x_{i}\right) \sim N\left(y_{i} \mid \mathbf{h}\left(x_{i}\right)^{T} \mathbf{w}+f\left(x_{i}\right), \sigma^{2}\right)
$$

\section{VARIABLE IMPORTANCE}

Variable importance is the technique which allows for the determination of the significance of each explanatory variable in reference to its effect on the implemented machine learning model [35]-[36]. This concept consists of the determination of the model error by permutations of the values of each variable. The given variable is significant if changes in its value cause changes in the model error, as the model relied on this variable

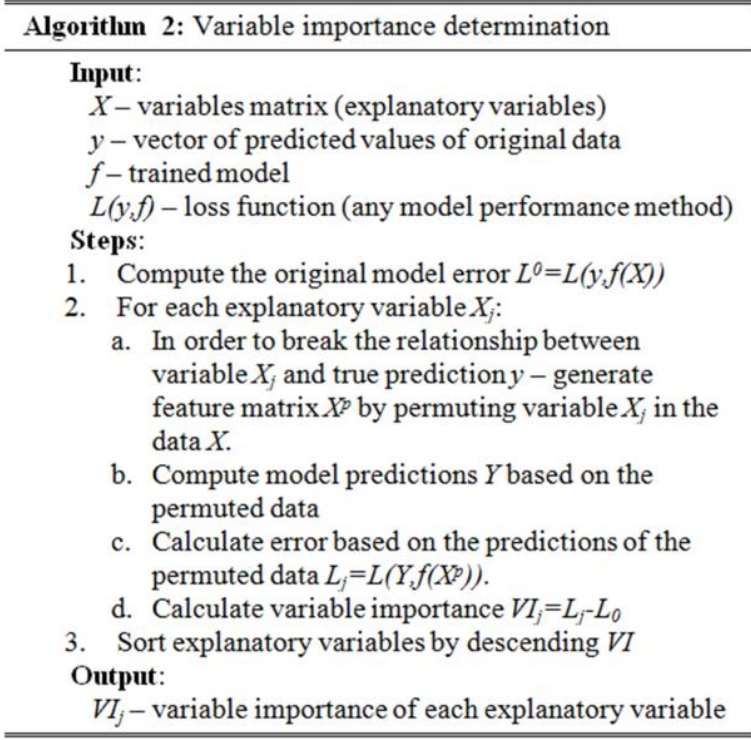

for prediction. If the permutations of the variable do not cause changes in the model error, then it is considered insignificant. In other words, the more the model relies on a variable to make predictions, the more important it is for the model. The values of significance of the variables are determined on the basis of algorithm 2 .

\section{ACCUMULATED LOCAL EFFECTS}

Accumulated local effects (ALE) is the method which allows for the assessment of the relationship between the explanatory variables of the model and the predicted value [36]-[37]. ALE reflects the way in which model forecasts change within the narrow range of values of variables. This method is particularly useful in the case of sets whose parameters are correlated with each other. The ALE function is defined as follows:

$$
\begin{aligned}
& \operatorname{ALE}\left(x_{1}\right)= \\
& \int_{\min \left(x_{1}\right)}^{x_{1}} E\left[\frac{\partial f\left(x_{1}, x_{2}\right)}{\partial x_{1}} \mid x_{1}=z_{1}\right] d z_{1}+c_{1}=
\end{aligned}
$$

where partial derivative $\frac{\partial f\left(x_{1}, x_{2}\right)}{\partial x_{1}}$ represents the local effect of $x_{1}$ on prediction function $f$ at $\left(x_{1}, x_{2}\right), \min \left(x_{1}\right)$ is lower value of considered variable range, $c_{l}$ is a constant selected in order to vertically center the graph.

Averaging the local effect over the conditional distribution $p\left(x_{2}, x_{1}\right)$ makes it possible to separate the effect of other correlated variables located outside the considered range of values of variable $x_{l}$.

In order to determine local effects, the whole range of values of the given explanatory variable must be divided into multiple intervals. The "effect" is understood as the difference in prediction calculated separately for each occurrence of a variable in a given interval. All differences in the given interval are summed up and divided by the number of occurrences - this way, the obtained mean difference in predictions is the "local" effect. Ultimately the value of the 

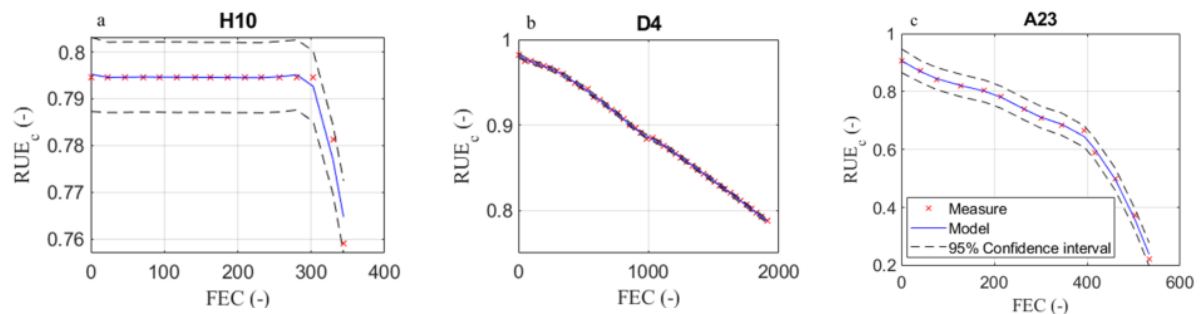

Fig 4. Learning results obtained for three observed types of $R U E_{c}$ evolution. (a) After about $300 F E C s$, the cell lost the ability to transfer the fixed energy - the phase of significant decrease in $R U E_{c}$ takes place $\left(M A P E=0.025 \%\right.$ ). (b) Under these conditions, throughout the lifetime of the LIC, the decrease in $R U E_{c}$ was approximately linear $(M A P E=0.003 \%)$. (c) The phase of slow degradation occurs until approximately $400 F E C s$ are completed, then it is possible to observe the phase of accelerated ability to transfer $R U E_{c}(M A P E=0.176 \%)$.

accumulated local effect of a given variable is determined by summing up all the local effects and represents the average change in the prediction when the value of the variable changes within its range.

The ALE method also allows for analysis of changes in the predicted value in the case of interaction of two (ALE secondorder) or more variables (ALE higher-order). In this case, the main impact of the variables concerned is ignored - only the effect of their interaction is calculated. For two explanatory variables, the ALE function may be represented as

$$
\begin{aligned}
& \operatorname{ALE}\left(x_{1}, x_{2}\right)= \\
& \int_{\min \left(x_{1}\right)}^{x_{1}} \int_{\min \left(x_{2}\right)}^{x_{2}} E\left[\frac{\partial f\left(x_{1}, x_{2}, x_{\{1,2\}}\right)}{\partial x_{1} \partial x_{2}} \mid x_{1}=z_{1}, x_{2}=z_{2}\right] d z_{1} d z_{2}
\end{aligned}
$$

The rules for the determination of ALE for two variables are identical to those for one variable, whereby, the intervals are replaced by rectangular areas due to the necessity of accumulation of local effects in two dimensions.

\section{RESULTS AND DISCUSSION}

The input parameters of the model were the cyclic operating parameters: the average LIC temperature during the full duty cycle $\left(T_{c}\right)$, the value of current during the discharge half-cycle $\left(I_{d}\right)$, depth of discharge $(D o D)$, the value of mean current during the charge half-cycle $\left(I_{\text {ch_avg }}\right)$ and the number of full equivalent cycles $(F E C S)$. The predicted value was the relative useful energy $\left(R U E_{c}\right)$ throughput of a cell during a full duty cycle.

\section{A. MODEL TRAINING}

Before initiating the machine learning procedure, the data obtained from the experimental measurements were normalised. After the normalisation, the average value of each of the parameters of the learning dataset was equal to 0 , and the standard deviation was equal to 1 . The results of measurements of cycling of LICs, which were cycled with $D o D>70 \%$ (20 LICs in total), were used as the learning dataset of the non-parametric GPR model. Cells "A38", "B32", "B40" and "H9" were used to verify the model exclusively. Finally, the learning dataset contained 311 samples, and the verification set consisted of 99 samples. The value of $F E C=0$ is equivalent to $\mathrm{SoH}=100 \%$ of the cell, while the last value of $F E C=533$ indicates $\mathrm{SoH}=80 \%$ and the

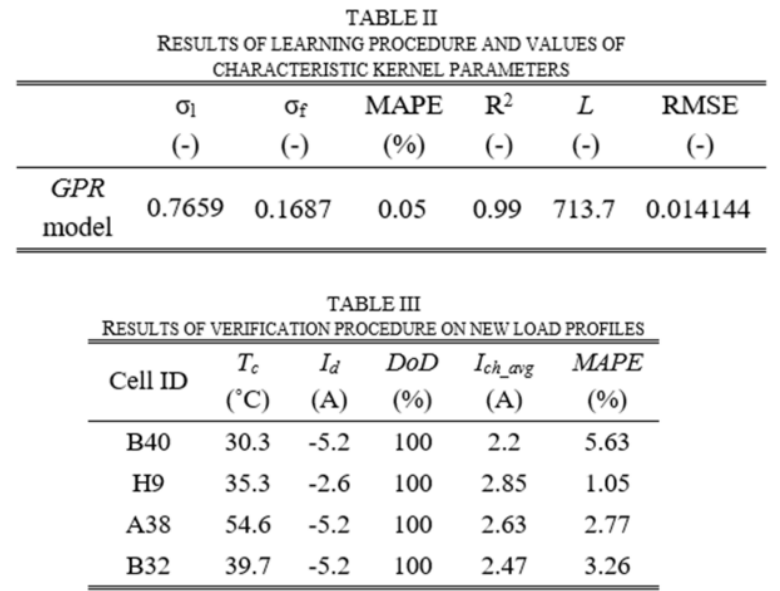

accomplishment of the EoL status. As the kernel function of the GPR model, the matern $3 / 2$ function was used, which is expressed by the following equation:

$$
k_{\text {mat } 3 / 2}\left(x, x^{\prime}\right)=\sigma_{f}^{2}\left(1+\frac{\sqrt{3} r}{\sigma_{l}}\right) \exp \left(-\frac{\sqrt{3} r}{\sigma_{l}}\right)
$$

where $r$ is Euclidean distance between $x$ and $x$.

The values of characteristic parameters $\sigma_{f}$ and $\sigma_{l}$ were determined using the method of optimisation without constraints (quasi-Newton).

Linear function type was adopted for the function set $h(x)$. The coefficient of determination $R^{2}$, mean average percentage error $M A P E$ and root mean square error (RMSE) were used to assess the model quality.

The results of the learning process of the GPR model: the values of the respective characteristic parameters are listed in Table II, while the example characteristics of the three observed types of $R U E_{c}$ evolution are presented in Fig. 4 (a-c). The learning results of other LICs are included in Appendix A. For each LIC included in the learning dataset, the response of the model was within the adopted confidence interval (95\%). The MAPE of the model developed for all samples of the learning dataset was $0.05 \%$. This proves that the GPR model almost perfectly matches the learning dataset.

\section{B. PREDICTION}

The developed original model was verified using new load profiles which were not used to train the model. LICs with different mean cycling temperatures $\left(T_{c}\right)$ at mean load current of $I_{d}=-5.2 \mathrm{~A}$ were selected for verification. For instance, for $T_{c}>40^{\circ} \mathrm{C}$, the learning dataset included LICs loaded with 

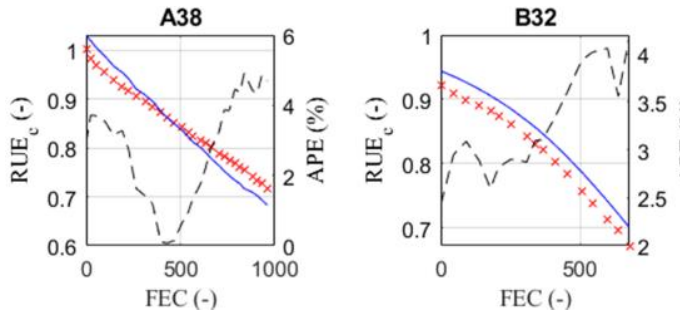

Fig. 5. Results of the model verification procedure.

$I_{d}=-2.6 \mathrm{~A}$ and $I_{d}=-7.8 \mathrm{~A}$ (cells "D6" and "H12"), therefore, the "A38" cell was selected for verification at higher temperatures. The verification process consisted of the $R U E_{c}$ prediction by a model of 4 cells in the period between $B o L$ and $E o L$, and of comparison of their values obtained during the experiment. The verification results are presented in Table III, and the characteristics for the 4 tested cases are presented in Fig. 5.

In the author's opinion, the obtained results of the verification process confirm the effectiveness of the selected GPR method for the purpose of $R U E_{c}$ prediction which a LIC can transfer during a full duty cycle throughout its life. Only in one test case did the MAPE slightly exceed 5\% (the "B40" cell). Also in favour of the method used, is the fact that despite an almost ideal matching of the model to the learning data, the prediction of $R U E_{c}$ under new operating conditions for the LIC is possible with little error. The GPR model also allows for prediction within ranges exceeding the ranges of variables of the learning dataset, which is not possible for some techniques based on artificial intelligence - for instance, neural networks or fuzzy logic.

\section{Model explanation}

In order to determine the significance of the explanatory variables of the model, the variable importance (VI) technique was used. RMSE was selected as the loss function of $L$ model. The calculations were repeated 8 times in order to negate the effect of uncertainty caused by permutations of values. The obtained results of VI are characterised by a low standard deviation; they were averaged and listed in Table IV. Based on the results, the respective model input parameters were ordered in terms of their significance - the effect of FEC on the predicted value of $R U E_{c}$ is the highest in the used dataset (the highest VI), then the effect of $I_{d}$ and $T_{c}$ is on a comparable level. From among the adopted model parameters, $D o D$ and $I_{\text {ch_avg }}$ have the lowest effect on $R U E_{c}$ (in both cases the calculated significance is at a similar level).

Given the fact that interpretation of the structure of the developed GPR non-parametric model is very difficult, in order to determine the effect which the respective model parameters have on the value of the $R U E_{c}$ prediction, the author has applied the ALE concept. Fig. 6 (a-e) presents the plots of the first-order accumulated local effects which determine the impact of individual input parameters of the GPR model on $R U E_{c}$. The value of ALE at the given point determines the difference in prediction in relation to the mean prediction of $R U E_{c}$. ALE $=0$ represents the mean prediction of $R U E_{c}$. For instance, for $T_{c} \approx 25^{\circ} \mathrm{C}$, the $R U E_{c}$ prediction will be lower by 0.05 in relation to the mean prediction which is an
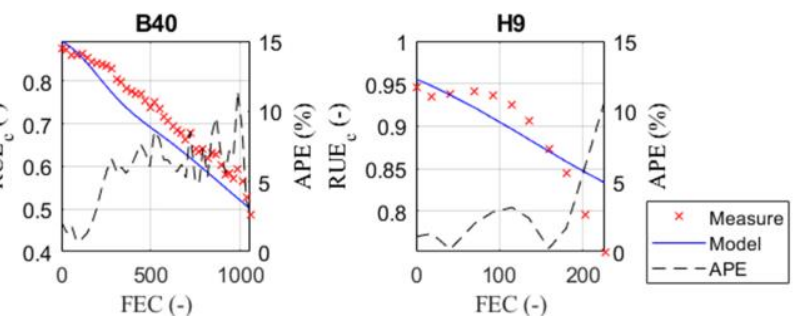

TABLE IV

VALUES OF VARIABLES IMPORTANCE OF IMPLEMENTED MODEL

\begin{tabular}{cccccc}
\hline \hline Parameter & $\begin{array}{c}T_{c} \\
\left({ }^{\circ} \mathrm{C}\right)\end{array}$ & $\begin{array}{c}I_{d} \\
(\mathrm{~A})\end{array}$ & $\begin{array}{c}D o D \\
(\%)\end{array}$ & $\begin{array}{c}I_{\text {ch__arg }} \\
(\mathrm{A})\end{array}$ & $\begin{array}{c}F E C \\
(-)\end{array}$ \\
\hline mean $V I$ & 0.120042 & 0.147175 & 0.048705 & 0.056989 & 0.167583 \\
std. dev. & 0.001375 & 0.001651 & 0.000866 & 0.000554 & 0.001614 \\
\hline \hline
\end{tabular}
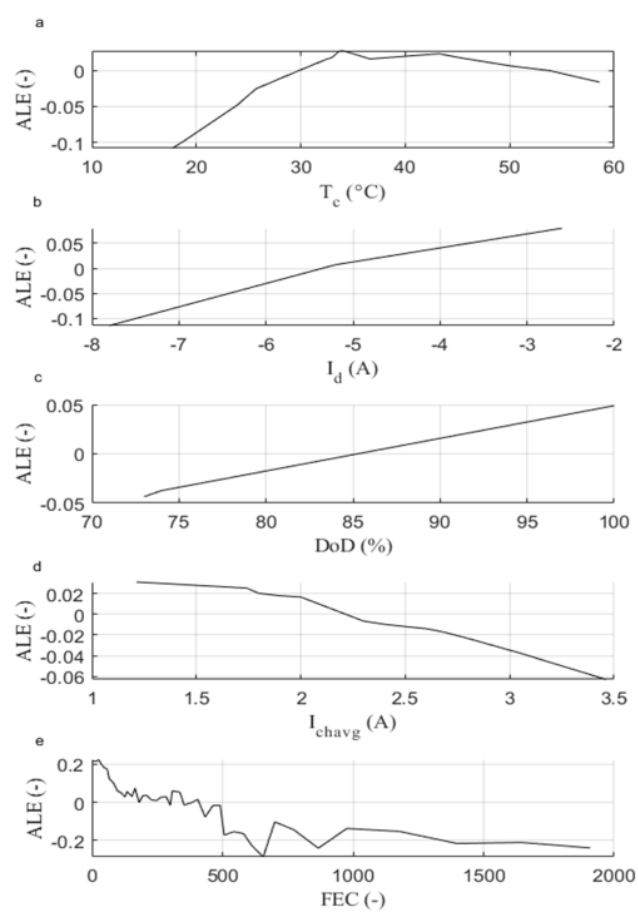

Fig. 6. First-order ALE plots for individual model input parameters (a) average temperature of LIC during the full cycle (b) discharge current (c) depth of discharge (d) average charge current (e) full equivalent cycle.

exclusive consequence of temperature (the effect of other cyclic operating parameters is separated).

Based on the results obtained, it is possible to determine, unambiguously, the effect of $T_{c}$ on $R U E_{c}$ - the lowest values of $R U E_{c}$ are obtained at average cycling temperatures below $30^{\circ} \mathrm{C}$ and they increase gradually up to the range of values between $30^{\circ} \mathrm{C}$ and $44^{\circ} \mathrm{C}$, where $R U E_{c}$ reaches its peak value and then falls for $T_{c}$ higher than $44^{\circ} \mathrm{C}$ (Fig. $6-$ a). In the case of values of currents $I_{d}$ and $I_{\text {ch_avg }}$, the effect on $R U E_{c}$ is linear. The lower the values of $I_{d}$ and $I_{c h \_a v g}$ are, the higher the $R U E_{c}$. is. Furthermore, cycling of the LICs at $I_{d}$ values lower than $5.2 \mathrm{~A}$ and higher than $2.25 \mathrm{~A}$ for $I_{\text {ch_avg }}$ will result in lower $R U E_{c}$ values over their lifetime - the visible breaking point of characteristics (Fig. $6-\mathrm{b}$, d). The plot of ALE for $D o D$ showed the linear dependence of this factor on $R U E_{c}$ (Fig. $6-\mathrm{c}$ ). The amount of transferred $R U E_{c}$ drops sharply when the cells complete more than 500 full equivalent duty 

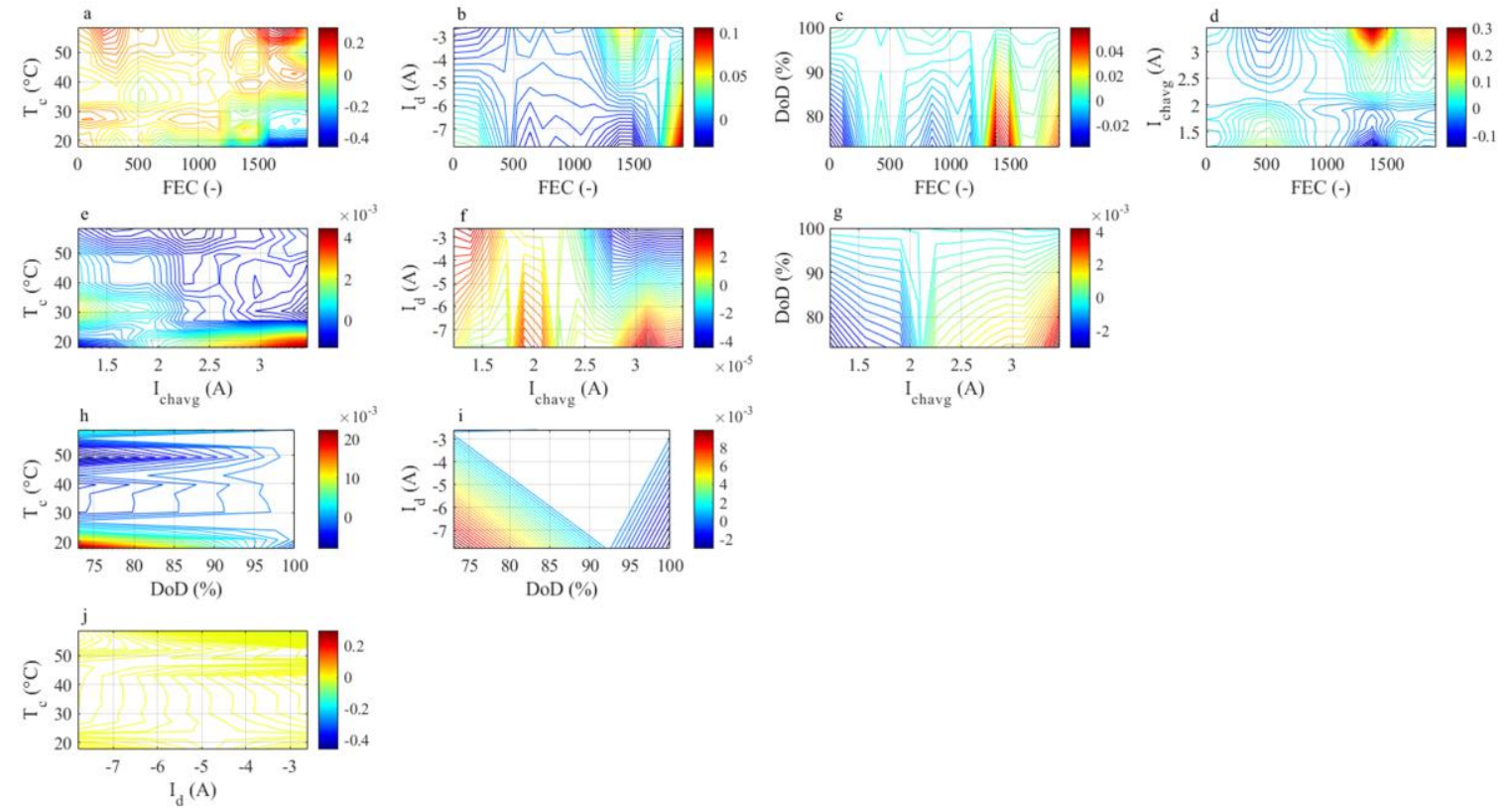

Fig. 7. Plots of accumulated local effects of the second order for the respective combinations of pairs of model input parameters.

cycles (Fig. $6-$ e).

Additionally, the results obtained using the ALE method confirm the results obtained using the VI technique - the highest dispersion of ALE values (from -0.286 to 0.225) is characteristic of $F E C$, which is compliant with the highest value of VI for this parameter. For $I_{d}$ and $T_{c}$ the differences in relation to the mean prediction vary from -0.114 to 0.08 for $I_{d}$ and from -0.108 to 0.027 for $T_{c}$ respectively, which also correlates with the VI results. The lowest dispersion of values was observed for $I_{\text {ch_avg }}-$ from -0.063 to 0.031 , which means the lowest effect on $R U E_{c}$ in the used dataset.

In order to determine the effect of interaction of the model parameters on $R U E_{c}$, the values of the second-order accumulated local effects were determined in the paper for all the possible combinations of pairs of the GPR model input parameters. The results obtained in the form of value maps are presented in Fig. 7 (a-j). Analysis of the interaction effect of model parameter pairs showed that the highest influence on $R U E_{c}$ is exerted by combinations of the following pairs $T_{c}-F E C, I_{d}-F E C$ and $I_{c h \_a v g}-F E C$. For instance, for LICs, which completed a large number of FECs (over 1500) at $T_{c}$ below $25^{\circ} \mathrm{C}$, the differences in relation to the mean $R U E_{c}$ prediction even amount to -0.4 , and at $T_{c}$ above $50^{\circ} \mathrm{C}+0.2$ respectively (Fig. 7 a). A high level of influence was also shown by the $I_{\text {ch_avg }}-F E C$ pair at around $F E C=1350$ and $I_{\text {ch_avg }}=3 \mathrm{~A}-$ the ALE value in this area reaches up to +0.3 (Fig. 7 d).The level of influence of the $D o D-F E C$ pair over the entire value range does not exceed the range between -0.04 and +0.06 (Fig. 7 d). To sum up - the pairs containing FEC are characterised by the highest influence due to the highest effect of $F E C$ on $R U E_{\mathrm{c}}$ which is shown in Fig. $6-\mathrm{e}$. The interaction effect of parameter pairs, i.e. $T_{c}-D o D$ and $T_{c}-I_{d}$ is locally at the level of hundredths (+0.02 and -0.015$)-$ Fig. 7 $-\mathrm{h}, \mathrm{j}$. On the other hand, the effect of other pairs is at a negligible level (ALE at a level of thousandths or lower) Fig. 7 - e-g,i.

\section{APPENDIX}

A. RESULTS OF THE LEARNING PROCEDURE

\begin{tabular}{cccccc}
\hline \hline Cell ID & $\begin{array}{c}\text { MAPE } \\
(\%)\end{array}$ & Cell ID & $\begin{array}{c}\text { MAPE } \\
(\%)\end{array}$ & Cell ID & $\begin{array}{c}\text { MAPE } \\
(\%)\end{array}$ \\
\hline D14 & 0.00424 & B35 & 0.036 & C26 & 0.0088 \\
A35 & 0.1015 & A30 & 0.1034 & D2 & 0.0149 \\
A20 & 0.0219 & B37 & 0.1902 & A25 & 0.0393 \\
D16 & 0.0262 & A31 & 0.2897 & D6 & 0.0095 \\
C10 & 0.0009 & H5 & 0.232 & C33 & 0.02779 \\
A23 & 0.1761 & D4 & 0.0031 & H12 & 0.00789 \\
H10 & 0.02456 & H1 & 0.03494 & & \\
\hline \hline
\end{tabular}

\section{REFERENCES}

[1] R. Pietracho et al., "Potential of Using Medium Electric Vehicle Fleet in a Commercial Enterprise Transport in Germany on the Basis of RealWorld GPS Data," Energies, vol. 14(17), pp. 1-23, 2021.

[2] K. Edströmet al., "BATTERY 2030+ Roadmap," p. 83, 2020.

[3] Z. Chen, Y. Ren, A. N. Jansen, C. K. Lin, W. Weng, and K. Amine, "New class of nonaqueous electrolytes for long-life and safe lithiumion batteries," Nat. Commun., vol. 4, pp. 1-8, 2013.

[4] M. Broussely et al., "Main aging mechanisms in Li ion batteries," $J$. Power Sources, vol. 146, no. 1-2, pp. 90-96, 2005.

[5] J. Vetter et al., "aging mechanisms in lithium-ion batteries," J. Power Sources, vol. 147, no. 1-2, pp. 269-281, 2005.

[6] A. Wang, S. Kadam, H. Li, S. Shi, and Y. Qi, "Review on modeling of the anode solid electrolyte interphase (SEI) for lithium-ion batteries," npj Comput. Mater., vol. 4, no. 1, 2018.

[7] J. Olmos, I. Gandiaga, A. Saez-de-Ibarra, X. Larrea, T. Nieva, and I. Aizpuru, "Modelling the cycling degradation of Li-ion batteries: Chemistry influenced stress factors," J. Energy Storage, vol. 40, no. May, p. 102765, 2021.

[8] M. Ecker et al., "Development of a lifetime prediction model for lithium-ion batteries based on extended accelerated aging test data," $J$. Power Sources, vol. 215, pp. 248-257, 2012.

[9] W. Diao, S. Saxena, and M. Pecht, "Accelerated cycle life testing and capacity degradation modeling of LiCoO2-graphite cells," J. Power Sources, vol. 435, no. June, p. 226830, 2019.

[10] M. Naumann, F. Spingler, and A. Jossen, "Analysis and modeling of cycle aging of a commercial LiFePO4/graphite cell," J. Power Sources, vol. 451, no. December 2019, p. 227666, 2020. 
[11] L. Tao, J. Ma, Y. Cheng, A. Noktehdan, J. Chong, and C. Lu, “A review of stochastic battery models and health management," Renew. Sustain. Energy Rev., vol. 80, no. February 2018, pp. 716-732, 2017.

[12] R. Xiong, Y. Zhang, J. Wang, H. He, S. Peng, and M. Pecht, "LithiumIon Battery Health Prognosis Based on a Real Battery Management System Used in Electric Vehicles," IEEE Trans. Veh. Technol., vol. 68, no. 5, pp. 4110-4121, 2019.

[13] S. N. Motapon, E. Lachance, L.-A. Dessaint, and K. Al-Haddad, "A Generic Cycle Life Model for Lithium-Ion Batteries Based on Fatigue Theory and Equivalent Cycle Counting," IEEE Open J. Ind. Electron. Soc., vol. 1, no. August, pp. 207-217, 2020.

[14]B. Saha, K. Goebel, S. Poll, and J. Christophersen, "Prognostics methods for battery health monitoring using a Bayesian framework," IEEE Trans. Instrum. Meas., vol. 58, no. 2, pp. 291-296, 2009.

[15] K. Liu, Y. Shang, Q. Ouyang, and W. D. Widanage, "A Data-Driven Approach with Uncertainty Quantification for Predicting Future Capacities and Remaining Useful Life of Lithium-ion Battery," IEEE Trans. Ind. Electron., vol. 68, no. 4, pp. 3170-3180, 2021.

[16]S. A. Hasibet al., "A Comprehensive Review of Available Battery Datasets, RUL Prediction Approaches, and Advanced Battery Management," IEEE Access, vol. 9, pp. 86166-86193, 2021.

[17] R. R. Richardson, C. R. Birkl, M. A. Osborne, and D. A. Howey, "Gaussian Process Regression for in Situ Capacity Estimation of Lithium-Ion Batteries," IEEE Trans. Ind. Informatics, vol. 15, no. 1, pp. 127-138, 2019.

[18] G. dos Reis, C. Strange, M. Yadav, and S. Li, "Lithium-ion battery data and where to find it," Energy AI, vol. 5, no. May, 2021.

[19] C. Vidal, P. Malysz, P. Kollmeyer, and A. Emadi, "Machine Learning Applied to Electrified Vehicle Battery State of Charge and State of Health Estimation: State-of-the-Art," IEEE Access, vol. 8, pp. 5279652814, 2020.

[20] D. Burzyński and L. Kasprzyk, "A novel method for the modeling of the state of health of lithium-ion cells using machine learning for practical applications," Knowledge-Based Syst., vol. 219, p. 106900, 2021.

[21]L. Kasprzyk, A. Domeracka, D. Burzyński, „Modelowanie pracy i trwałości akumulatorów litowo-jonowych w pojazdach elektrycznych," Przegląd Elektrotechniczny., no. 12, 160-163, 2018.

[22] D. N. T. How, M. A. Hannan, M. S. H. Lipu, K. S. M. Sahari, P. J. Ker, and K. M. Muttaqi, "State-of-Charge Estimation of Li-Ion Battery in Electric Vehicles: A Deep Neural Network Approach,” IEEE Trans. Ind. Appl., vol. 56, no. 5, pp. 5565-5574, 2020.

[23] Y. Li, K. Li, X. Liu, and L. Zhang, "Fast battery capacity estimation using convolutional neural networks," Trans. Inst. Meas. Control, 2020.

[24] I. H. Li, W. Y. Wang, S. F. Su, and Y. S. Lee, “A merged fuzzy neural network and its applications in battery state-of-charge estimation," IEEE Trans. Energy Convers., vol. 22, no. 3, pp. 697-708, 2007.

[25] X. Hu, J. Jiang, D. Cao, and B. Egardt, "Battery health prognosis for electric vehicles using sample entropy and sparse Bayesian predictive modeling," IEEE Trans. Ind. Electron., vol. 63, no. 4, pp. 2645-2656, 2016.
[26]D. Burzyński, R. Pietracho, L. Kasprzyk, and A. Tomczewski, "Analysis and Modeling of the Wear-Out Process of a Lithium-NickelManganese-Cobalt Cell during Cycling Operation under Constant Load Conditions," Energies, vol. 12, no. 20, p. 3899, Oct. 2019.

[27]A. El Mejdoubi, H. Chaoui, H. Gualous, P. Van Den Bossche, N. Omar, and J. Van Mierlo, "Lithium-ion batteries health prognosis considering aging conditions," IEEE Trans. Power Electron., vol. 34, no. 7, pp. 6834-6844, 2019.

[28] R. R. Richardson, M. A. Osborne, and D. A. Howey, "Battery health prediction under generalized conditions using a Gaussian process transition model," J. Energy Storage, vol. 23, no. March, pp. 320-328, 2019.

[29]P. Tagade et al., "Deep Gaussian process regression for lithium-ion battery health prognosis and degradation mode diagnosis," J. Power Sources, vol. 445, no. October 2019, p. 227281, 2020.

[30] K. Liu, Y. Li, X. Hu, M. Lucu, and W. D. Widanage, "Gaussian Process Regression with Automatic Relevance Determination Kernel for Calendar Aging Prediction of Lithium-Ion Batteries," IEEE Trans. Ind. Informatics, vol. 16, no. 6, pp. 3767-3777, 2020.

[31]R. Xiong, Y. Zhang, H. He, X. Zhou, and M. G. Pecht, "A doublescale, particle-filtering, energy state prediction algorithm for lithiumion batteries," IEEE Trans. Ind. Electron., vol. 65, no. 2, pp. 1526$1538,2017$.

[32]K. Li, F. Wei, K. J. Tseng, and B. H. Soong, “A Practical Lithium-Ion Battery Model for State of Energy and Voltage Responses Prediction Incorporating Temperature and aging Effects," IEEE Trans. Ind. Electron., vol. 65, no. 8, pp. 6696-6708, 2018.

[33]C. E. Rasmussen and Ch. K. Williams, "Gaussian processes for machine learning, "MIT press Cambridge, vol. 1, 2006.

[34] S. Haykin and S. Simon, "Neural Networks and learning machines," Prentice Hall, New York, 2009.

[35]P. Wei, Z. Lu, and J. Song, "Variable importance analysis: A comprehensive review," Reliab. Eng. Syst. Saf., vol. 142, pp. 399-432, 2015.

[36]C. Molnar, "Interpretable Machine Learning. A Guide for Making Black Box Models Explainable.," Book, p. 247, 2019.

[37] D. W. Apley and J. Zhu, "Visualizing the effects of predictor variables in black box supervised learning models," J. R. Stat. Soc. Ser. B Stat. Methodol., vol. 82, no. 4, pp. 1059-1086, 2020.

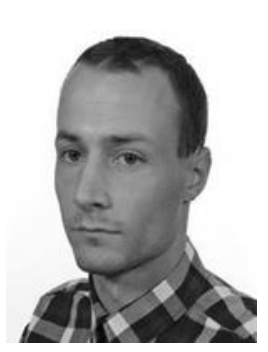

Damian Burzynski Received his MSc degree in electrical engineering from the Poznan University of Technology in 2015. $\mathrm{He}$ is an assistant lecturer and is also working towards his $\mathrm{PhD}$ degree at the Poznan University of Technology. His research interests cover modelling the operation and cycle life of electrochemical batteries using non-deterministic methods. 